\title{
Thermodynamics driving the strong metal-support interaction: titanate encapsulation of supported Pd nanocrystals
}

\author{
Peiyu Chen, ${ }^{* \dagger}$ Yakun $\mathrm{Gao}^{\dagger}$ and Martin R. Castell*
}

Department of Materials, University of Oxford, Parks Road, Oxford, OX1 3PH, United Kingdom

*Corresponding authors: peiyu.chen@materials.ox.ac.uk, martin.castell@materials.ox.ac.uk

${ }^{\dagger}$ These authors contributed equally to this work.

Abstract: The strong metal-support interaction (SMSI) is the encapsulation of a supported metal particle by an oxide layer that diffuses from the substrate. This process is usually described as being driven by a reduction in the surface energy of the metal particle and has a significant influence on the catalytic activity of the metal. Here, epitaxial Pd nanocrystals grown in ultrahigh vacuum on $\mathrm{SrTiO}_{3}(001)$ and anatase $\mathrm{TiO}_{2}(001)$ substrates are studied by scanning tunneling microscopy. At annealing temperatures above $\sim 600{ }^{\circ} \mathrm{C}$, the $\mathrm{Pd}$ crystals can become encapsulated by a $\mathrm{TiO}_{x}$ monolayer originating from the substrates. For both bare and encapsulated Pd crystals, their height-to-width ratio increases with the crystal height as a mechanism to partially release their interfacial misfit strain with the substrate. However, the rate of this increase is lower for encapsulated crystals, indicating that during the SMSI process the interface between the particle and the oxide is modified to form a lower energy interfacial structure which also results in less strain in the encapsulated particle. The SMSI is found to preferentially occur on larger crystals, driven by the reduction in their elastic strain energy, which scales with the crystal volume. Compared with the traditional view of SMSI our results provide a more complete description of the encapsulation process. 


\section{Introduction}

Palladium (Pd) and other noble metal nanocrystals supported on oxide substrates are popular materials systems in heterogeneous catalysis. For example, $\mathrm{Pd}$ is used in automotive catalytic converters and formic acid fuel cells. The interaction between Pd crystals and titanium oxide supports has been the focus of a number of studies over the past twenty years [1-7]. Under reducing conditions [e.g., in ultrahigh vacuum (UHV)] and elevated temperatures (usually $400-700{ }^{\circ} \mathrm{C}$ ), a thin layer of reduced $\mathrm{Ti}$ suboxide, $\mathrm{TiO}_{x}(1 \leq x \leq 1.5)$, can migrate from the substrate onto the metal nanocrystal surface [2-11]. This is due to the strong metal-support interaction (SMSI), where the thermodynamic driving force for encapsulation is usually described as a lowering of the metal crystal surface energy [12]. Encapsulation significantly influences the catalytic activity of the metal nanocrystals $[6,7,10,11]$.

$\mathrm{TiO}_{x}$ encapsulation can be identified using scanning tunneling microscopy (STM) and/or low energy electron diffraction (LEED) by the observation of moiré superstructures between the $\mathrm{TiO}_{x}$ overlayer and the metal surface, usually on $\mathrm{Pd}(111)[2,4,13]$ and $\mathrm{Pt}(111)$ surfaces $[3,8,9]$. In contrast to the relatively easily observed encapsulation on the outer surface of metal particles, only a few studies have conjectured that the metal-oxide support interface was also modified during SMSI $[14,15]$. It has also been reported that no change in the crystal structure was observed for the $\mathrm{Pd}-\mathrm{TiO}_{2}$ interface when encapsulation occurred [16], although this study strictly speaking only confirms that the interface was not changed to the extent that the crystallography or epitaxial orientation was affected. The interaction and bonding between the metal and the support can also significantly affect the catalytic behavior of the sites at the rim of the metal-support interface [17-20].

In this paper, we report a study of encapsulated Pd crystals grown on single crystal strontium titanate $\left(\mathrm{SrTiO}_{3}\right)(001)$ substrates (nanostructured or terminated with an anatase film). Pd crystals have a face- 
centered-cubic (fcc) structure and their Wulff shape is a truncated octahedron terminated by $\{111\}$ and $\{001\}$ facets. On our substrates, equilibrium Pd crystals adopt a (111) interface with the substrates (Fig. 1) [2,21-23]. The Pd crystals become encapsulated by $\mathrm{TiO}_{x}$ resulting from the substrates when heated at $\sim 600{ }^{\circ} \mathrm{C}$ or above, as indicated schematically by the blue overlayer on the orange crystal in Fig. 1(b). The encapsulated crystals display clear moiré patterns on their top facets under STM imaging. Their aspect ratio also becomes flatter than for their bare crystal counterparts, which, according to the traditional SMSI model, is due to surface energy reduction [12].

(a)
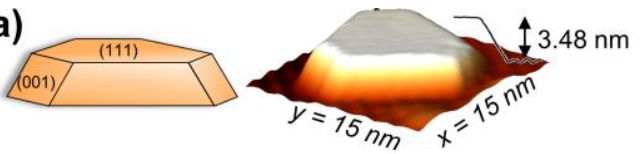

(b)

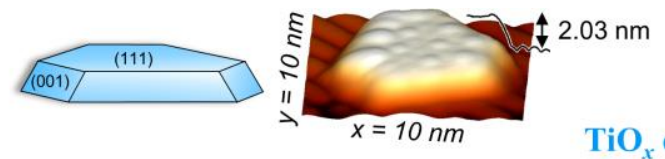

(c)

(111)

(d)

(111) $\mathrm{TiO}_{x}$ overlayer

\begin{abstract}
(e)
\end{abstract}

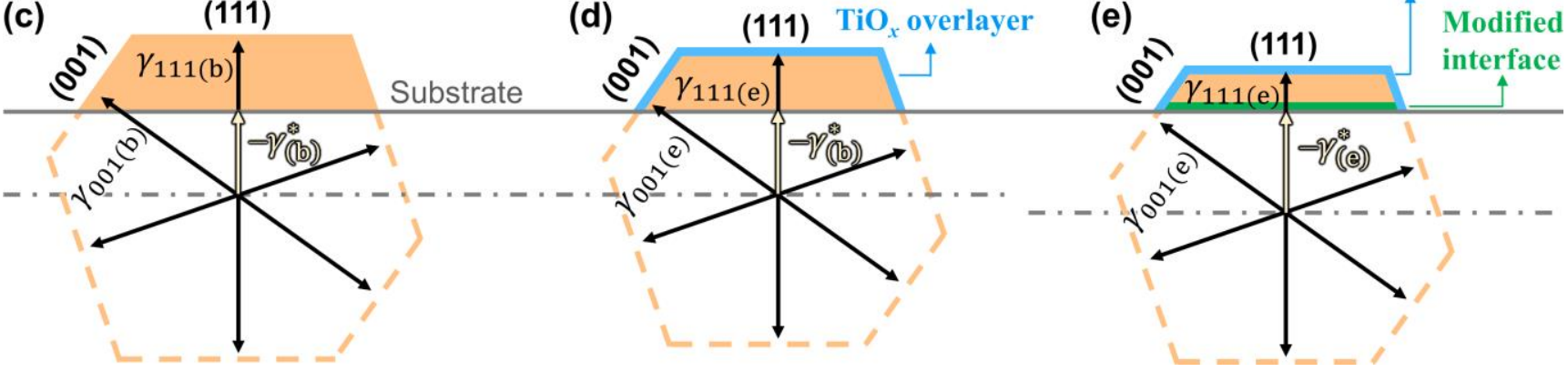

FIG. 1. Bare and encapsulated Pd crystals. (a,b) 3D sketches and 3D-rendered STM images of bare (a) and encapsulated (b) Pd crystals. (c-e) Winterbottom constructions of substrate-supported bare (c) and encapsulated (d,e) Pd crystals. The model in (d) shows a $\mathrm{TiO}_{x}$ overlayer (blue) encapsulating the crystal outer surface and the model in (e) also includes a modified interface (green).

As sketched in the Winterbottom constructions [24,25] in Figs. 1(c) and 1(d), upon encapsulation, the facet energies of $\mathrm{Pd}, \gamma_{111(\mathrm{~b})}$ and $\gamma_{001(\mathrm{~b})}$, are reduced in magnitude and are replaced by $\gamma_{111(\mathrm{e})}$ and $\gamma_{001(\mathrm{e})}$, where the subscripts "b" and "e" refer to "bare" and "encapsulated", respectively. In fact, $\gamma_{111(\mathrm{e})}$ is the sum of the surface energy of the $\mathrm{TiO}_{x}$ overlayer and the interfacial energy between $\mathrm{TiO}_{x}$ and $\operatorname{Pd}(111)$, and similarly for $\gamma_{001(e)}$. The energy $\gamma^{*}$ is the difference between the interfacial energy and the substrate surface energy $\left(\gamma^{*}=\gamma_{\mathrm{i}}-\gamma_{\mathrm{s}}\right)$ [24]. In the traditional SMSI model, the crystal-substrate interface is unchanged, and therefore we have $\gamma_{(b)}^{*}$ in both Figs. 1(c) and 1(d). However, by measuring 
the crystal shape change, we deduce that during SMSI, in addition to the energy reductions on the $\{111\}$ and $\{001\}$ facets, the interfacial energy and interfacial strain must also have been reduced, where the modified interface is shown in green in Fig. 1(e). This leads to a change in $\gamma^{*}$ where $-\gamma_{(\mathrm{e})}^{*}>-\gamma_{(\mathrm{b})}^{*}$ in Fig. 1(e). Our result leads to a significant revision of the traditional view of the outcome of the SMSI process in that we show that both the metal crystal outer surface and its interface are modified.

\section{Experimental methods}

$\mathrm{SrTiO}_{3}$ is a cubic perovskite crystal which exhibits a variety of surface reconstructions under different sample preparation conditions. Our $\mathrm{SrTiO}_{3}$ samples were epi-polished on the (001) surface and doped with $\mathrm{Nb}$ at $0.5 \%$ by weight, supplied by PI-KEM, U.K. On $\mathrm{SrTiO}_{3}(001)$, we prepared two surfaces: the nanostructured surface covered with dilines and trilines [26-28] and the anatase $\mathrm{TiO}_{2}(001)$ epitaxial thin film structure with a $(1 \times 4)$ reconstruction [29,30]. The nanostructured surface [Fig. 2(a)] was produced by $\mathrm{Ar}^{+}$-ion sputtering at $1.0 \mathrm{keV}$ for $10 \mathrm{~min}$ and subsequent annealing in $\mathrm{UHV}$ at $860{ }^{\circ} \mathrm{C}$ for $1.5 \mathrm{~h}$ [26-28]. To generate the anatase thin film [Fig. 2(b)], a total of 3 monolayers of Ti was deposited onto nanostructured $\mathrm{SrTiO}_{3}(001)$ in five cycles from an $e$-beam evaporator (Oxford Applied Research EGCO4) using 99.8+\% pure Ti rods supplied by Advent, U.K. After each Ti deposition, the sample was post-annealed at $850{ }^{\circ} \mathrm{C}$ for $1.5-5 \mathrm{~h}$, totaling $12 \mathrm{~h}$. As shown in Fig. 2, the prepared substrates were crystalline, atomically flat, and relatively free of contaminants, which are critical to our later analysis of epitaxial strain between the Pd crystals and the two substrate surfaces. 

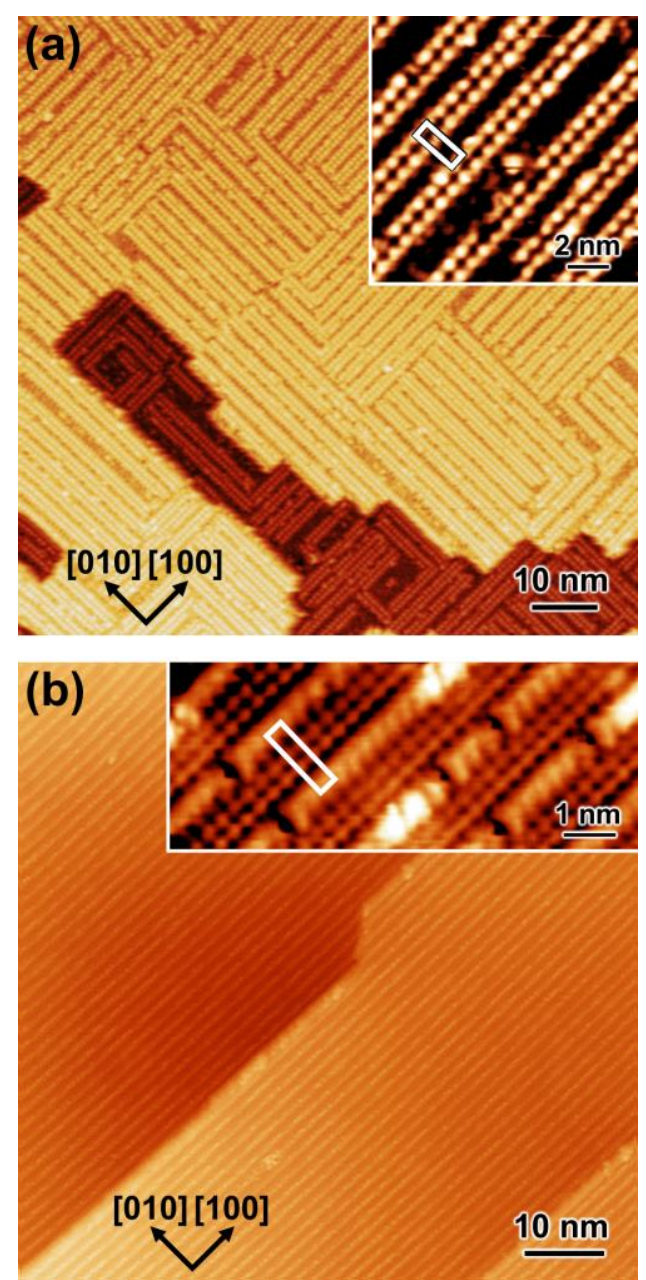

FIG. 2. STM images of the (a) nanostructured $\mathrm{SrTiO}_{3}(001)$ and (b) anatase substrates. The insets are higher-magnification images with rectangular unit cells drawn $\left(\mathrm{a}: V_{\mathrm{s}}=1.9 \mathrm{~V}, I_{\mathrm{t}}=0.24 \mathrm{nA}\right.$; inset: $V_{\mathrm{s}}=$ $1.8 \mathrm{~V}, I_{\mathrm{t}}=0.18 \mathrm{nA}$; b: $V_{\mathrm{s}}=1.6 \mathrm{~V}, I_{\mathrm{t}}=0.23 \mathrm{nA}$; inset: $V_{\mathrm{s}}=1.3 \mathrm{~V}, I_{\mathrm{t}}=0.60 \mathrm{nA}$ ). The crystallographic lattice directions of $\mathrm{SrTiO}_{3}(001)$ are labeled.

On the nanostructured $\mathrm{SrTiO}_{3}(001)$ and anatase substrates, $\mathrm{Pd}$ was deposited from the $e$-beam evaporator using $99.95 \%$ pure Pd rods supplied by Goodfellow, U.K. The Pd crystals were postannealed in UHV at $\sim 450{ }^{\circ} \mathrm{C}$ or above, which is necessary for the crystals to reach their equilibrium shapes and hence an accurate representation of their surface/interface energies [21]. The prepared substrates and Pd crystals were imaged by STM (JEOL JSTM 4500s model, base pressure $10^{-8} \mathrm{~Pa}$ ). STM images were processed using Gwyddion and WSxM [31]. 


\section{Results and discussion}

As shown in the 3D-rendered STM images in Fig. 3, equilibrium Pd crystals have top facets with a truncated triangular shape, on both the nanostructured $\mathrm{SrTiO}_{3}(001)$ [Fig. 3(b)] and anatase [Figs. 3(a,c)] substrates. The crystals tend to align one of their $\{111\}$ edge facets with the $<100>$ substrate directions on both substrates, so the interfacial crystallography can be described as $(111)_{\mathrm{Pd}} \|(001)_{\mathrm{SrTiO}_{3}}$, $[1 \overline{1} 0]_{\mathrm{Pd}} \|[100]_{\mathrm{SrTiO}_{3}}$. This arrangement allows near-perfect coincidence epitaxy between 7 Pd unit cells $(\mathrm{Pd}-\mathrm{Pd}$ bond length $=2.751 \AA)$ and 5 substrate unit cells: on $\mathrm{SrTiO}_{3}(001), 7 \times 2.751 \AA \approx 5 \times 3.905 \AA$, with a $+1.4 \%$ tensile strain in $\mathrm{Pd}$; on anatase, $7 \times 2.751 \AA \approx 5 \times 3.785 \AA$, with a $-1.8 \%$ compressive strain in Pd. Such favorable coincidence lattice registry has been confirmed to be a powerful driver in determining the epitaxial alignment of supported crystals on crystalline substrates [32-34]. Also, we expect the misfit strain levels here $(+1.4 \%$ and $-1.8 \%)$ to be accommodated elastically on both substrates.

Figure 3(a) shows some Pd crystals post-annealed at $550{ }^{\circ} \mathrm{C}$ for $2 \mathrm{~h}$, exhibiting a consistent shape with very similar top-facet shapes and crystal aspect ratios. The crystals tend to decorate high-energy sites on the substrate, which in Fig. 3(a) are terrace steps and emerging screw dislocations. When the crystals are post-annealed at $\sim 600^{\circ} \mathrm{C}$ or above, some crystals become encapsulated by a thin $\mathrm{TiO}_{x}$ film originating by diffusion from the substrate. For example, Fig. 3(b) shows some crystals post-annealed at $650^{\circ} \mathrm{C}$ for $1 \mathrm{~h}$, among which the four crystals indicated by white arrows are encapsulated, exhibiting a wagon wheel moiré superstructure with a periodicity of $19.0 \pm 0.5 \AA$ [2]. A bare crystal and an encapsulated crystal are magnified in Figs. 3(c.i) and 3(c.ii), respectively. It can be seen from Fig. 3(b) that in general, encapsulated crystals have a flatter aspect ratio than bare ones. For example, the height profiles are drawn for a bare crystal and an encapsulated crystal: their top facets are similar in 

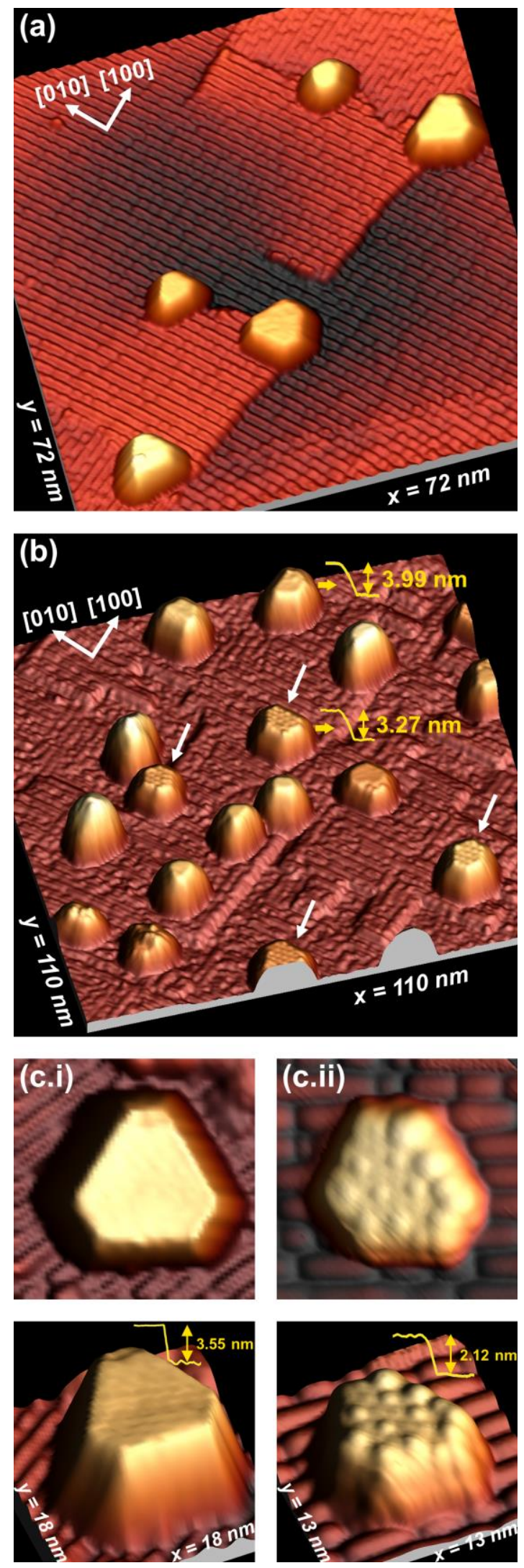

FIG. 3. 3D-rendered STM images of Pd crystals. (a) Pd crystals nucleated at $300{ }^{\circ} \mathrm{C}$ and post-annealed at $550{ }^{\circ} \mathrm{C}$ for $2 \mathrm{~h}$ on anatase $\left(V_{\mathrm{s}}=1.6 \mathrm{~V}, I_{\mathrm{t}}=0.17 \mathrm{nA}\right)$. (b) $\mathrm{Pd}$ crystals nucleated at $650{ }^{\circ} \mathrm{C}$ and postannealed at $650{ }^{\circ} \mathrm{C}$ for $1 \mathrm{~h}$ on nanostructured $\mathrm{SrTiO}_{3}(001)\left(V_{\mathrm{s}}=2.0 \mathrm{~V}, I_{\mathrm{t}}=0.30 \mathrm{nA}\right)$. Some crystals are encapsulated by a $\mathrm{TiO}_{x}$ overlayer, showing a wagon wheel superstructure on the top facet, indicated by arrows. (c) Magnified images of a bare (c.i) and an encapsulated (c.ii) crystal on anatase: top view (upper row) and oblique view (bottom row) (c.i: $V_{\mathrm{s}}=2.0 \mathrm{~V}, I_{\mathrm{t}}=0.17 \mathrm{nA}$; e.ii: $V_{\mathrm{s}}=2.6 \mathrm{~V}, I_{\mathrm{t}}=1.33 \mathrm{nA}$ ). 
size, but the bare crystal is $\sim 20 \%$ higher $(3.99 \mathrm{~nm})$ than the encapsulated one $(3.27 \mathrm{~nm})$.

Also, we observe that about $58 \%$ of the Pd crystals become encapsulated after being annealed at 600 $650{ }^{\circ} \mathrm{C}$, while all are encapsulated at $860^{\circ} \mathrm{C}$. We attribute this difference to the kinetic energy available for diffusion at the different temperatures.

(a)
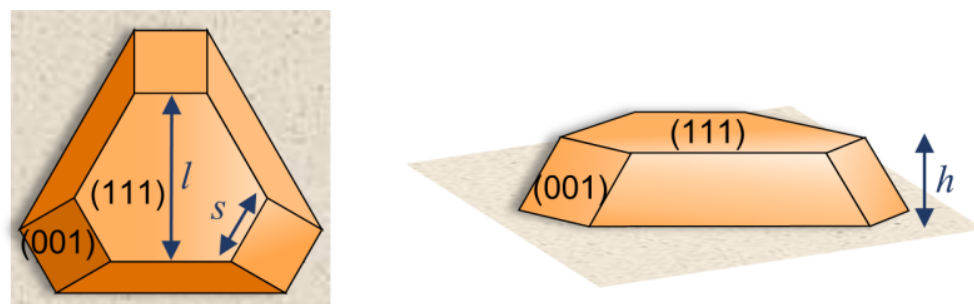

(b)

- Bare crystals

$\Delta$ Encapsulated crystals

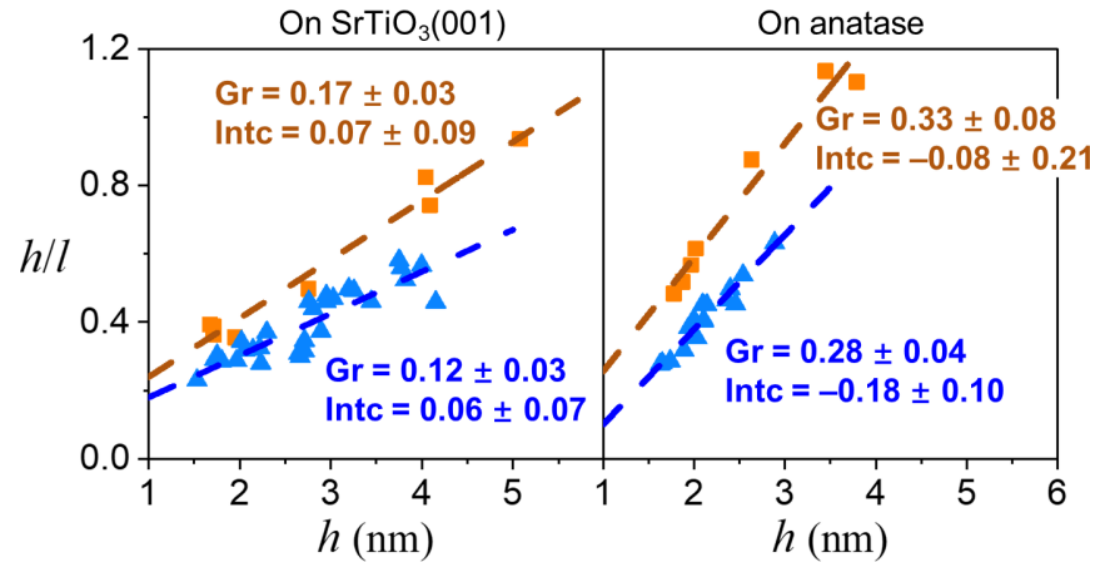

FIG. 4. Aspect ratio of Pd crystals. (a) Top and oblique views of the Wulff shape of a supported Pd crystal, with three dimensions labeled: $l, s$, and $h$. (b) $h / l$ plotted against $h$ for Pd crystals on nanostructured $\mathrm{SrTiO}_{3}(001)$ and anatase substrates.

The thermodynamics of the energy minimization of the Pd crystals can be analyzed by measuring the change in crystal shape. We measured three dimensions labeled in the sketches in Fig. 4(a): $l$ is the width across the top (111) facet measured from the middle of one $\{001\}$ side facet to the middle of the opposite $\{111\}$ side facet; $s$ is the width of a $\{001\}$ facet; $h$ is the crystal height. These dimensions were only measured from well-equilibrated $\mathrm{Pd}$ crystals that were post-annealed at $\geq 450{ }^{\circ} \mathrm{C}$, are three-fold rotationally symmetric, and have flat top facets. To obtain reliable height measurements, we also 
excluded crystals attached to step edges [e.g., as in Fig. 3(a)] because their base facets are not flat. Out of thousands of crystals that we imaged, only $\sim 60$ satisfy all the equilibrium criteria above.

The $s / l$ ratio is a measure of the relative areas of the $\{111\}$ and $\{001\}$ facets, and is related to the facet energies, $\gamma_{111}$ and $\gamma_{001}$, via

$$
\frac{\gamma_{111}}{\gamma_{001}}=\frac{1}{\sqrt{3}}+\frac{s}{2 l}
$$

The Pd crystals were found to have a constant $s / l$ ratio with crystal size, which is $0.39 \pm 0.04$ for both bare and encapsulated crystals, and is independent of the substrate. Substituting $s / l=0.39 \pm 0.04$ into equation (1), we obtain $\frac{\gamma_{111(\mathrm{~b})}}{\gamma_{001(\mathrm{~b})}}=0.77 \pm 0.02[21]$ and $\frac{\gamma_{111(\mathrm{e})}}{\gamma_{001(\mathrm{e})}}=0.77 \pm 0.02$.

The $h / l$ ratio of bare and encapsulated Pd crystals is plotted against the crystal height $h$ on nanostructured $\mathrm{SrTiO}_{3}(001)$ and anatase substrates in Fig. 4(b). In all cases, there is a positive relationship between $h / l$ and $h$, as shown by the fitted linear regression lines. This is because the crystals heighten to partially release the interfacial strain caused by the lattice mismatch at the Pd-substrate interface. Within a Pd crystal, the strength of the strain field is expected to be the strongest at the interface and decays as it moves away from the interface, as discussed in our previous work on Au crystals [34]. The overall strain field strength depends on the misfit strain at the crystal-substrate interface.

The thermodynamic reason for crystal heightening is demonstrated schematically in Fig. 5. For a crystal epitaxially supported on a lattice-mismatched substrate, its total energy consists of the formation enthalpy (negative), the surface/interface energies (positive), and the strain energy due to the interfacial misfit (positive) [35-37]. In our current analysis we do not include the entropy terms. With a given volume, the crystal has a fixed formation enthalpy regardless of its shape, which is therefore neglected from now on. The other two energy terms are qualitatively sketched in Fig. 5. 


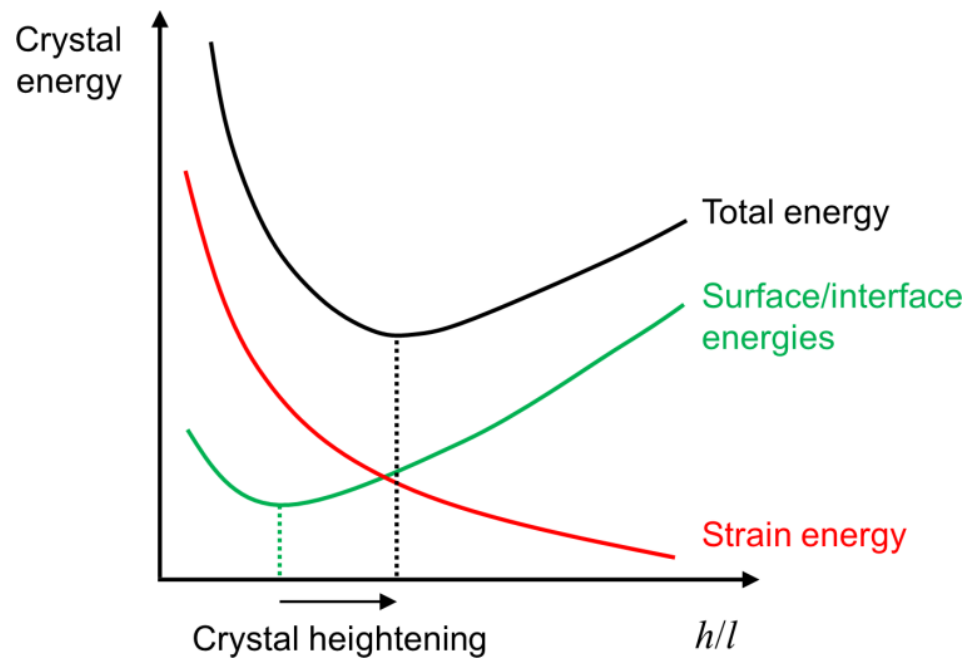

FIG. 5. Qualitative sketches of the energy components of a crystal with a constant volume supported on a lattice-mismatched substrate, illustrating the origin of strain-induced crystal heightening.

If the supported crystal is strain-free, its equilibrium $h / l$ ratio is predicted by the Winterbottom construction, where a substrate cuts a free-standing Wulff crystal to minimize its total surface/interface energies [24,25]. Accordingly, the $h / l$ ratio should be a constant for a given substrate, and it is related to $\gamma_{111}, \gamma_{001}$, and $\gamma^{*}$ via $[38]$

$$
\frac{h}{l}=\sqrt{\frac{2}{3}} \frac{\gamma^{*}+\gamma_{111}}{\gamma_{001}}
$$

This equilibrium $h / l$ ratio corresponds to the minimum on the green curve in Fig. 5 (surface/interface energies), indicated by the green dashed line.

Strain generated at the interface due to epitaxial lattice mismatch results in an elastic energy term. Its functional form is a complicated expression that depends on a number of factors including the crystal and substrate stiffness, the lattice misfit between them, the crystal volume, and the crystal shape [36,39]. It is sketched schematically in red in Fig. 5, where the strain energy reduces with an increasing $h / l$ ratio, approaching zero as $h / l \rightarrow \infty$. This is because a higher aspect ratio for a given volume results in more exposed sidewall of the crystal, which is where elastic strain relaxation can take place. The presence of 
the strain energy term upshifts the equilibrium value of $h / l$, as indicated by the minimum on the total energy curve in black. This explains the observed crystal heightening in Fig. 4(b), which is a mechanism for strain relief.

In Fig. 4(b), the gradients for the $h / l$ plots are greater on anatase than on nanostructured $\mathrm{SrTiO}_{3}(001)$ for both bare and encapsulated crystals, due to the larger misfit strain at the Pd-anatase interface $(-1.8 \%)$ than at the $\mathrm{Pd}-\mathrm{SrTiO}_{3}(001)$ interface $(+1.4 \%)$. We note that these misfit strains are only in the direction of $[1 \overline{1} 0]_{\mathrm{Pd}} \|[100]_{\mathrm{SrTiO}_{3}}$. However, one-dimensional coincidence epitaxy was shown to generate interfacial strain and manipulate the crystal aspect ratio in the same way as two-dimensional epitaxy, albeit less powerfully [34]. On both substrates the $h / l$ ratio is smaller for the encapsulated crystals than for the bare crystals at a given height $h$, consistent with the STM observation in Fig. 3(b). In addition, the $h / l$ gradient is shallower for encapsulated crystals than for bare crystals, suggesting that the epitaxial misfit strain must have been reduced following the SMSI process. This means that the interfacial structure has transformed to achieve both a lower interfacial energy and a reduction in interfacial strain. Similar differences in $h / l$ gradients were also observed for bare Au nanocrystals supported on different $\mathrm{SrTiO}_{3}$ substrates in our previous work [34]. No encapsulation was observed so the different crystal aspect ratios were solely attributed to the different interfacial energies and interfacial strain.

The traditional view of the SMSI process is that only the crystal surface facets are affected. However, based on our measurements here, we propose that a structural change also occurs at the metal-support interface. It has been shown that at high temperatures, the encapsulation of metal particles starts with the diffusion of Ti cations towards the surface of the oxide substrate [40]. It would not be surprising if, as well as an encapsulating layer forming on the outer surface of the metal particle, there is also a structural change at the metal-support interface which brings about a reduction in interfacial energy. 
Although the epitaxial relationship between Pd crystals and the substrates remains the same after encapsulation [Fig. 3(a)], this does not provide evidence of an unchanged interface - it only means that the interface was not changed to the extent that the interfacial crystallography was affected.

Using the change in $h / l$ ratio of the encapsulated Pd crystals, we can estimate their change in surface/interface energies. Rearranging equation (2), we can write for bare and encapsulated Pd crystals:

$$
\begin{aligned}
& \gamma_{(\mathrm{b})}^{*}=\sqrt{\frac{3}{2}}\left(\frac{h}{l}\right)_{\mathrm{b}} \gamma_{001(\mathrm{~b})}-\gamma_{111(\mathrm{~b})}, \\
& \gamma_{(\mathrm{e})}^{*}=\sqrt{\frac{3}{2}}\left(\frac{h}{l}\right)_{\mathrm{e}} \gamma_{001(\mathrm{e})}-\gamma_{111(\mathrm{e})} .
\end{aligned}
$$

We start by assuming that SMSI only affects the Pd surface facets but not the Pd-substrate interface, so $\gamma_{(\mathrm{b})}^{*}=\gamma_{(\mathrm{e})}^{*}$. Within our experimentally observed crystals, those which are the least strained at the interface are the smallest crystals (e.g., $h<2 \mathrm{~nm}$ ) on nanostructured $\mathrm{SrTiO}_{3}(001)$. We therefore use these crystals to estimate the strain-free equilibrium $h / l$ ratios, and we obtain $\left(\frac{h}{l}\right)_{\mathrm{b}}=0.37 \pm 0.02$ for bare crystals and $\left(\frac{h}{l}\right)_{\mathrm{e}}=0.28 \pm 0.03$ for encapsulated crystals. Using $\gamma_{111(\mathrm{~b})}=0.77 \gamma_{001(\mathrm{~b})}$ and $\gamma_{111(\mathrm{e})}=$ $0.77 \gamma_{001(\mathrm{e})}$ from our results above, and using $\gamma_{111}=1.77 \mathrm{~J} \mathrm{~m}^{-2}$ following Patra et al. [41], we calculate $\gamma_{111(\mathrm{e})}=1.29 \pm 0.16 \mathrm{~J} \mathrm{~m}^{-2}$ and $\gamma_{001(\mathrm{e})}=1.67 \pm 0.20 \mathrm{~J} \mathrm{~m}^{-2}$. It follows that the facet energy reductions if one considers only surface encapsulation are $\Delta \gamma_{111}=-0.48 \pm 0.16 \mathrm{~J} \mathrm{~m}^{-2}$ and $\Delta \gamma_{001}=$ $-0.61 \pm 0.21 \mathrm{~J} \mathrm{~m}^{-2}$

The values of these energy reductions are larger than expected. As an indication, Pt covered by a monolayer of $\mathrm{Ti}_{2} \mathrm{O}_{3}$ is associated with an energy reduction of $\sim-0.2 \mathrm{~J} \mathrm{~m}^{-2}$ according to DFT calculations [42]. This suggests that the change in the Pd crystal shape cannot be attributed to encapsulation alone, but that the interfacial structure has also changed. Thus, instead of equating 
equations (3) and (4), we subtract them from one another and obtain a linear relationship between $\gamma_{(\mathrm{e})}^{*}$ and $\gamma_{111(\mathrm{e})}$ :

$$
\gamma_{(\mathrm{e})}^{*}=\left\{\frac{\gamma_{(\mathrm{b})}^{*}}{\gamma_{111(\mathrm{~b})}}-\frac{1}{0.77} \sqrt{\frac{3}{2}}\left[\left(\frac{h}{l}\right)_{\mathrm{b}}-\left(\frac{h}{l}\right)_{\mathrm{e}}\right]\right\} \gamma_{111(\mathrm{e})}=-0.56 \gamma_{111(\mathrm{e})} .
$$

If we replace $\gamma_{(\mathrm{e})}^{*}$ by $\gamma_{(\mathrm{b})}^{*}+\Delta \gamma^{*}$ and $\gamma_{111(\mathrm{e})}$ by $\gamma_{111(\mathrm{~b})}+\Delta \gamma_{111}$, and substitute in the numerical values for the flattest crystals $(h<2 \mathrm{~nm})$ on nanostructured $\mathrm{SrTiO}_{3}(001)$, it follows that $\Delta \gamma^{*}$ and $\Delta \gamma_{111}$ are related by

$$
\Delta \gamma^{*}=-0.56 \Delta \gamma_{111}-0.27
$$

Equation (6) is plotted in Fig. 6, which shows that to achieve the observed change in $h / l$, we can change either $\gamma_{111}$ by $-0.48 \mathrm{~J} \mathrm{~m}^{-2}$ or $\gamma^{*}$ by $-0.27 \mathrm{~J} \mathrm{~m}^{-2}$ or any combination in between. Note that this plot represents an upper bound of the energy reductions required, since the Pd crystals analyzed are not completely strain-free and the observed shape change is still partly due to strain relief during SMSI.

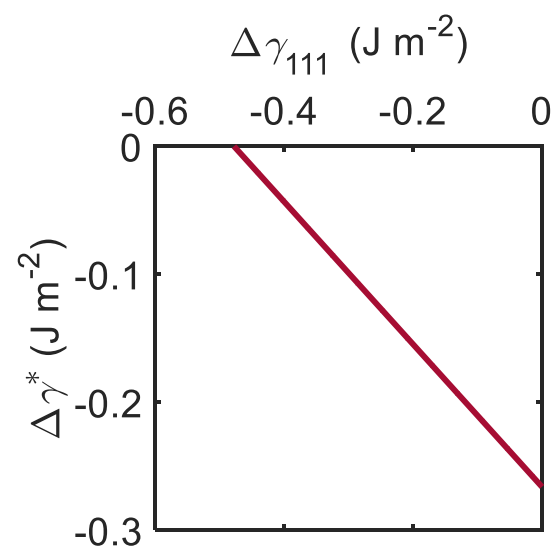

FIG. 6. $\Delta \gamma^{*}$ vs $\Delta \gamma_{111}$ for the flattest crystals $(h<2 \mathrm{~nm})$ on nanostructured $\mathrm{SrTiO}_{3}(001)$.

Finally, we observe that larger crystals are more likely to have been encapsulated. Figure 7 shows that for Pd crystals post-annealed at $\geq 600{ }^{\circ} \mathrm{C}$, there is a greater proportion of encapsulated crystals at larger 
crystal volumes. Such size-dependent SMSI observations have also be reported for $\mathrm{TiO}_{2}$-supported $\mathrm{Au}$ nanoparticles, where larger particles were preferentially encapsulated [43]. This size dependence is the opposite of what one would expect using kinetic arguments, since the encapsulation of larger crystals requires a greater amount of atomic diffusion. Therefore, this observation must be explained by a larger thermodynamic driving force: it is energetically more favorable to release the interfacial strain of larger crystals since the elastic strain energy stored in them scales with crystal volume. Again, the strain relief occurs via SMSI, during which process the atomic structure of the crystal-substrate interface is reconfigured and the crystal outer surface is encapsulated. We note that if the effect of larger crystals being preferentially encapsulated is strain driven, then this effect should disappear beyond a critical size where misfit dislocations at the interface relieve the bulk Pd crystal strain.

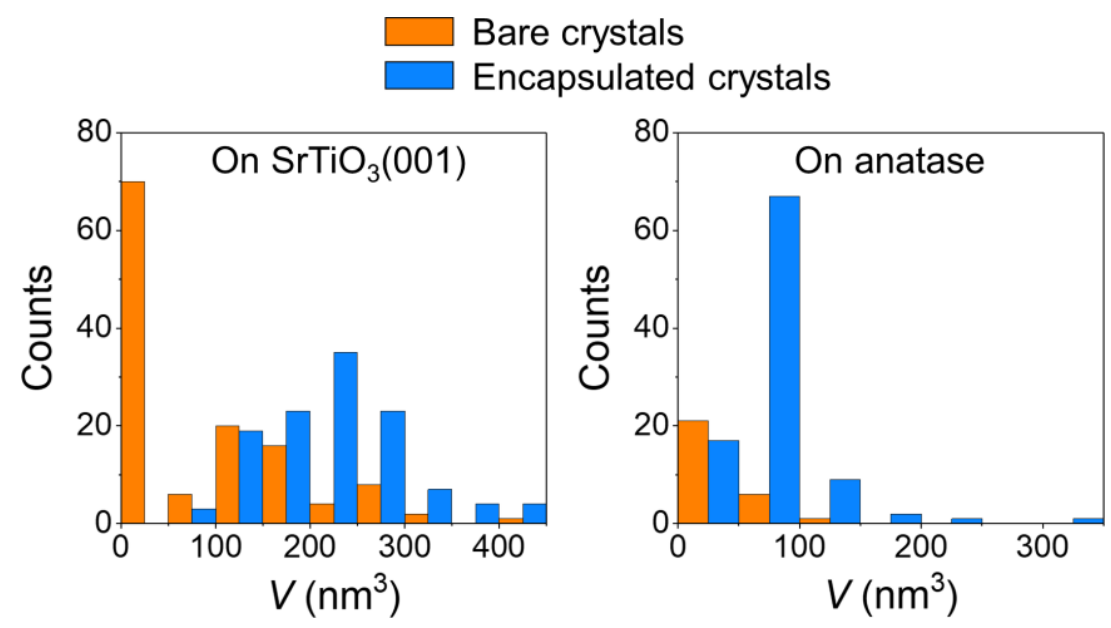

FIG. 7. Populations of bare and encapsulated Pd crystals that have been post-annealed at $\geq 600{ }^{\circ} \mathrm{C}$ on nanostructured $\mathrm{SrTiO}_{3}(001)$ and anatase substrates, plotted against crystal volume.

In real catalysts, there is not always an epitaxial relationship between the metal particles and their support, which means little strain relief during SMSI. However, an interfacial structural change will still take place, reducing the interfacial energy, though the metal particle shape change caused by this structural change alone may be insufficiently evident to be measured and studied. On the other hand, 
our use of single crystalline substrates, by introducing interfacial misfit strains, amplifies the modification of the interfacial structure and induces a larger shape change in the metal particles. It is this degree of amplification that allows us to infer that SMSI introduces an interfacial structural change in addition to encapsulation. Also, studying the crystals in UHV allows us to extract accurate information on their facet/interface energies, which lead to our important conclusions above. Otherwise, the crystals would be covered in adsorbents, and their shapes would reflect the surface energies of the absorbent-modified facets. These UHV results are relevant to real catalysts that do not operate under UHV as they also undergo SMSI, which is of interest because it significantly affects the catalytic activity of the noble metals.

\section{Conclusions}

In summary, $\mathrm{Pd}$ crystals were grown epitaxially on nanostructured $\mathrm{SrTiO}_{3}(001)$ and anatase $\mathrm{TiO}_{2}(001)$ substrates, with truncated triangular (111) top and base facets. After being annealed at $\geq 600{ }^{\circ} \mathrm{C}$, some crystals are encapsulated by a $\mathrm{TiO}_{x}$ thin film originating from the substrates, showing a wagon wheel moiré superstructure on their top facets. The equilibrium shaped Pd crystals show an increasing $h / l$ ratio with crystal height $h$ due to the misfit strain at their interface with the substrates. The rate at which the $h / l$ ratio increases with $h$ is lower for encapsulated crystals than for bare crystals, which is attributed to the less-strained and lower-energy interface of the encapsulated crystals as a result of SMSI. We also calculate that even for the least strained crystals in our study, their change in aspect ratio is too large to be explained by a reduction in surface energies alone, but a reduced interfacial energy is also required to justify the shape change. Therefore, we propose that in addition to the traditional view of the SMSI effect, the interfacial structure has been modified, leading to a lower interfacial energy and lower 
interfacial strain. This is also supported by our observation that SMSI preferably occurs on larger crystals, thermodynamically driven by the reduction in their elastic strain energy, which scales with the crystal volume. Our conclusion provides helpful insights into metal-support interaction and hence the lifetime of the activity of some important heterogenous metal particle catalyst materials systems.

\section{Acknowledgements}

This work was supported by EPSRC grants EP/K032518/1 and EP/M015173/1. The authors would like to thank Chris Spencer (JEOL U.K.) for technical support and the China Scholarship Council (CSC) for financial support.

\section{References}

[1] U. Diebold, Surf. Sci. Rep. 48, 53 (2003).

[2] F. Silly and M. R. Castell, J. Phys. Chem. B 109, 12316 (2005).

[3] R. A. Bennett, C. L. Pang, N. Perkins, R. D. Smith, P. Morrall, R. I. Kvon, and M. Bowker, J. Phys. Chem. B 106, 4688 (2002).

[4] M. Bowker, P. Stone, P. Morrall, R. Smith, R. Bennett, N. Perkins, R. Kvon, C. Pang, E. Fourre, and M. Hall, J. Catal. 234, 172 (2005).

[5] Y. Li, Y. Fan, H. Yang, B. Xu, L. Feng, M. Yang, and Y. Chen, Chem. Phys. Lett. 372, 160 (2003).

[6] P. Weerachawanasak, P. Praserthdam, M. Arai, and J. Panpranot, J. Mol. Catal. A Chem. 279, 133 (2008).

[7] Y. Li, B. Xu, Y. Fan, N. Feng, A. Qiu, J. M. J. He, H. Yang, and Y. Chen, J. Mol. Catal. A Chem. 216, 107 (2004).

[8] O. Dulub, W. Hebenstreit, and U. Diebold, Phys. Rev. Lett. 84, 3646 (2000). 
[9] D. R. Jennison, O. Dulub, W. Hebenstreit, and U. Diebold, Surf. Sci. 492, L677 (2001).

[10] S. J. Tauster, S. C. Fung, and R. L. Garten, J. Am. Chem. Soc. 100, 170 (1978).

[11] S. J. Tauster, Acc. Chem. Res. 20, 389 (1987).

[12] Q. Fu, T. Wagner, S. Olliges, and H.-D. Carstanjen, J. Phys. Chem. B 109, 944 (2005).

[13] M. Bowker and E. Fourré, Appl. Surf. Sci. 254, 4225 (2008).

[14] S. Liu, W. Xu, Y. Niu, B. Zhang, L. Zheng, W. Liu, L. Li, and J. Wang, Nat. Commun. 10, 5790 (2019).

[15] S. Bonanni, K. Aït-Mansour, H. Brune, and W. Harbich, ACS Catal. 1, 385 (2011).

[16] T. Suzuki and R. Souda, Surf. Sci. 448, 33 (2000).

[17] A. Namdeo, S. M. Mahajani, and A. K. Suresh, J. Mol. Catal. A Chem. 421, 45 (2016).

[18] G. Pacchioni, Phys. Chem. Chem. Phys. 15, 1737 (2013).

[19] Q. Fu, F. Yang, and X. Bao, Acc. Chem. Res. 46, 1692 (2013).

[20] J. Xu, K. Sun, L. Zhang, Y. Ren, and X. Xu, Catal. Commun. 6, 462 (2005).

[21] P. Chen, Y. Gao, and M. R. Castell, Appl. Phys. Lett. 117, 101601 (2020).

[22] T. Wagner, G. Richter, and M. Rüble, J. Appl. Phys. 89, 2606 (2001).

[23] B. R. Chen, C. George, Y. Lin, L. Hu, L. Crosby, X. Hu, P. C. Stair, L. D. Marks, K. R. Poeppelmeier, R. P. Van Duyne, and M. J. Bedzyk, Surf. Sci. 648, 291 (2016).

[24] W. L. Winterbottom, Acta Metall. 15, 303 (1967).

[25] R. Kaishew, Arbeitstagung Festkörper Physik (Dresden, 1952).

[26] D. S. Deak, F. Silly, D. T. Newell, and M. R. Castell, J. Phys. Chem. B 110, 9246 (2006).

[27] H. L. Marsh, D. S. Deak, F. Silly, A. I. Kirkland, and M. R. Castell, Nanotechnology 17, 3543 (2006).

[28] M. S. J. Marshall, A. E. Becerra-Toledo, D. J. Payne, R. G. Egdell, L. D. Marks, and M. R. Castell, Phys. Rev. B 86, 125416 (2012).

[29] F. Silly and M. R. Castell, Appl. Phys. Lett. 85, 3223 (2004).

[30] M. S. J. Marshall and M. R. Castell, Phys. Rev. Lett. 102, 146102 (2009).

[31] I. Horcas, R. Fernández, J. M. Gómez-Rodríguez, J. Colchero, J. Gómez-Herrero, and A. M. Baro, Rev. Sci. Instrum. 78, 013705 (2007).

[32] J. Sun, C. Wu, F. Silly, A. A. K. Koo, F. Dillon, N. Grobert, and M. R. Castell, Chem. Commun. 49, 3748 (2013). 
[33] P. Chen, W. Xu, Y. Gao, J. H. Warner, and M. R. Castell, ACS Appl. Nano Mater. 1, 6976-6988 (2018).

[34] P. Chen, K. Murugappan, and M. R. Castell, Phys. Chem. Chem. Phys. 22, 4416 (2020).

[35] G. Medeiros-Ribeiro, A. M. Bratkovski, T. I. Kamins, D. A. A. Ohlberg, and R. S. Williams, Science 279, 353 (1998).

[36] P. Müller and R. Kern, Surf. Sci. 457, 229 (2000).

[37] V. A. Shchukin and D. Bimberg, Rev. Mod. Phys. 71, 1125 (2002).

[38] F. Silly and M. R. Castell, Phys. Rev. Lett. 94, 046103 (2005).

[39] P. Müller and R. Kern, Microsc. Microanal. Microstruct. 8, 229 (1997).

[40] Q. Fu and T. Wagner, Surf. Sci. Rep. 62, 431 (2007).

[41] A. Patra, J. E. Bates, J. Sun, and J. P. Perdew, Proc. Natl. Acad. Sci. U. S. A. 114, E9188 (2017).

[42] J. Goniakowski and C. Noguera, J. Phys. Chem. C 124, 8186 (2020).

[43] X. Du, Y. Huang, X. Pan, B. Han, Y. Su, Q. Jiang, M. Li, H. Tang, G. Li, and B. Qiao, Nat. Commun. 11, 5811 (2020). 